\title{
Progress of Electromagnetic Compatibility Design for Unmanned Aerial Vehicles
}

\author{
Yong $\mathrm{Wu}^{1,2, a}$, Qishuang $\mathrm{Ma}^{1}$ and Ping $\mathrm{Xu}^{1}$ \\ ${ }^{1}$ College of Automation Science and Electrical Engineering, Beihang University ,Beijing 100191,China \\ ${ }^{2}$ Beijing ELectro-Mechanical Engineering Institute, China Aerospace Science and Industry Corporation,Beijing 100074,China
}

\begin{abstract}
The electromagnetic environment of UAV system was studied, and three key elements of electromagnetic interference(EMI) are analyzed. The research status of electromagnetic characteristics of UAV system at home and abroad was summarized from numerical simulation of EMI, experimental test of EMI and design of electromagnetic compatibility(EMC). The main contents include the research on electromagnetic characteristics of airborne antenna, EMI of data link and its mechanism, electromagnetic characteristics of internal cables, electromagnetic radiation test, electromagnetic conduction test, system-level EMC design, etc. The key technical challenges faced by the research on EMC of unmanned aerial vehicles at home and abroad are pointed out.
\end{abstract}

\section{Research Environment}

When UAV is flying, it is not only affected by electromagnetic environment caused by radiation of internal equipment, but also by external electromagnetic environment such as lightning, interference station, nuclear electromagnetic pulse and so on. If these interference can not be prevented reasonably, the performance of airborne equipment will be greatly affected $^{[1]}$.

Electromagnetic environment refers to the sum of power or field interference in different frequency range and the kinds and states of various interference sources when the UAV is performing its mission ${ }^{[2]}$. The external electromagnetic environment of UAV can be divided into natural electromagnetic environment and man-made electromagnetic environment. The internal electromagnetic environment is focused on here. The internal electromagnetic environment of UAV mainly includes antenna feed system, electromechanical system and circuit system.

\section{EMI and EMC}

EMI source, interference propagation path and sensitive equipment are called as three elements of EMI. Among them, EMI source refers to any component, equipment, system or natural phenomenon that produces interference, including intentional and unintentional emission; interference propagation means the medium that transmits EMI energy to sensitive equipment, which can be divided into conduction coupling and radiation coupling; sensitive equipment refers to the circuit and equipment that are susceptible to interference ${ }^{[3]}$.

EMC refers to the electromagnetic environment in which the equipment or system meets the requirements and does not produce unbearable EMI to other equipment. According to the definition, EMC needs to satisfy three conditions: the electromagnetic disturbance generated by equipment or system in normal operation can not cause the performance degradation of other equipment; the electromagnetic disturbance generated by other equipment in electromagnetic environment has a certain tolerance; the electromagnetic disturbance that can not affect its performance degradation. Generally speaking, EMC is a subject that studies the coexistence and normal operation of various devices or systems in a specific working environment and limited spectrum resources.

\section{Current Research Situation at Home and Abroad}

\subsection{Evaluation of EMC and its Criteria}

In the 1970s, EMC testing technology was studied abroad. In 1996, the European Union began to implement the EMC license system for products. At present, developed countries such as Europe and the United States have made fruitful achievements in EMC evaluation criteria and standards. In the 1980s, China began to systematically study the national and industrial standards and specifications of EMC. At present, more than 30 national and military standards have been formulated.

\footnotetext{
a Corresponding author: 34846717@qq.com
} 
The typical EMC evaluation system of UAV is shown in the evaluation criteria of UAV system EMC can be divided into two categories: system or equipment performance evaluation criteria based on deterministic electromagnetic environment characteristics and statistical analysis evaluation criteria based on stochastic environmental characteristics. Song Zuxun et al. [4] analyzed the evaluation criteria based on the characteristics of deterministic electromagnetic environment, but did not consider the EMI with obvious randomness. Zhu Zhiyu et al ${ }^{[5]}$ gave the EMC standard of transient pulse group resistance. Li Bo et al. ${ }^{[6]}$ obtained the magnetic field strength limit value of UAV system through the radiation and electromagnetic environment test of ground transmitter, and preliminarily formulated the EMC design evaluation criteria of UAV system. Zhang Yong et al ${ }^{[7]}$ determined the evaluation index system of ship EMC, which can be used to guide the design of evaluation criteria of UAV EMC. Li Xiaojian et al. [8] preliminarily established system level EMC evaluation indexes and performance evaluation criteria.

EMC evaluation criteria is the basis of UAV avionics system design. According to the previous research experience, in the future, empirical analysis prediction method and index comprehensive construction method should be used in the model development to analyze and study the specific index, and optimize the whole system, so as to finally pass the consistency verification of calculation analysis and test.

\subsection{Numerical Simulation of EMI of UAV}

\subsubsection{Study on Electromagnetic Characteristics of Airborne Antenna}

Airborne antenna layout is one of the EMC design of UAV. Inter-antenna coupling, antenna pattern and nearfield radiation have a great impact on the performance of airborne multi-antenna system, and are the main indicators to measure the layout of aircraft multi-antenna system. Among them, the coupling degree between antennas is the most important parameter to measure the EMC between antennas.

Tan Yuanyang ${ }^{[9]}$ predicted the installation location of antenna. So the installed antenna not only meets the aerodynamic requirements of aircraft, but also achieves their main technical and tactical indicators and EMC between antennas. Yuan Jun $^{[10]}$ adopted adaptive fast multipole algorithm to solve the linear structure, analyzed the EMC pattern, isolation, interference power and other important indicators of airborne antenna system. Chen Jinji ${ }^{[11]}$ proposed two methods for calculating antenna isolation. Eight airborne antennas were designed by HFSS simulation. Peng Tianhao ${ }^{[12]}$ analyzed the EMI caused by the radio frequency excitation source in the meter-wave broadband plasma antenna and the overload of the instrument, the harmonic distortion of the measuring equipment and the harmonic noise in the communication frequency band. Liu Baihan ${ }^{[13]}$ studied the numerical simulation technology of airborne antenna in radio frequency band on aircraft. Han Xuefeng ${ }^{[14]}$ obtained the antenna pattern affected by the airframe and the layout design, and through the analysis of the electromagnetic radiation characteristics of the airborne antenna, obtained the optimum electromagnetic field distribution and antenna distribution area.

\subsubsection{Research on EMI and its mechanism in data link}

UAV airborne receiver is composed of multistage amplifier, mixer and filter. For UAV upstream data link, if the interference signal intensity entering the airborne receiver is large enough to exceed the dynamic range of various electronic devices in the receiver, the sensitivity of the receiver will be reduced and the signal-to-noise ratio will be reduced, which can be called blocking interference $^{[15]}$.

UAV communication is highly dependent on data link and vulnerable to battlefield electromagnetic environment during its mission. In UAV data link system, the receiver is sensitive to $\mathrm{EMI}^{[16]}$. EMI will cause the non-linear distortion of airborne receivers, which will lead to the interruption of data link communication and seriously threaten the battlefield survival of UAVs.

Jiao Yanwei ${ }^{[17]}$ used cloud model method to study the comprehensive effectiveness of UAV in complex electromagnetic environment, and established the effectiveness evaluation index system of hierarchical structure of UAV in complex electromagnetic environment. Liu Ruiqi et al. ${ }^{[18]}$ deduced the inequality of tracking interference hyperboloid and limited power interference range from the time domain and power condition of tracking interference, determined the effective interference range under different parameters, and proposed a reasonable jamming start-up strategy. Guo Hongjing ${ }^{[19]}$ studied the cognitive strategy and cognitive process of UAV TT\&C link interference. Zhang Xiaodong et al. ${ }^{[20]}$ carried out the upstream main remote control lock-out sensitive frequency point injection method research, obtained the spectrum change rule of useful signal after interference, and obtained the lock-out mechanism through BPSK modulation and demodulation analysis. Guo Shuxia et al. ${ }^{[21]}$ developed a scene view method based on GRA and the dynamic realtime driving method of microwave instrument according to the performance of real-time simulation and the intelligent function of simulation system, and simulated the dynamic interference of static radiation antenna in microwave anechoic chamber. Shen Yan'an ${ }^{[22]}$ used Simulink simulation platform to build the UAV remote control link model based on direct spread spectrum technology. Song Zuxun et al. [23] calculated the interference probability or the number of received interference pulses, and compared with the fault-tolerant ability of the data chain.

\subsubsection{Numerical simulation of electromagnetic characteristics of internal cables}

Wire plays an important role in the EMC of UAV. Wires can be equivalent not only to receiving antennas, but also 
to transmitting antennas. According to statistics, the total weight of all kinds of cables on a large transport aircraft reaches several tons, and $60 \%$ of all kinds of EMI occurring on the aircraft is due to the electromagnetic coupling between cables.

Jiangdan $^{[24]}$ used three-dimensional electromagnetic simulation software CST to model and simulate the electromagnetic characteristics of cables inside the aircraft. Wang Chao ${ }^{[25]}$ modeled and simulated the electromagnetic coupling characteristics of aircraft interconnected cables. Tan Yujian ${ }^{[26]}$ studied the frequency domain solution method BLT equation and applied it to the analysis of transmission line crosstalk with arbitrary layout; also studied the time domain solution method-FDTD method, and deduced the iteration formula under lumped source loading and distributed source loading.

\subsection{EMI Test of Unmanned Aerial Vehicle}

\subsubsection{Measurement of electromagnetic radiation}

Radiation interference refers to the interference propagating in the form of electromagnetic waveform. The energy of this kind of interference is due to the radiation of the interference source, which propagates through the medium with the characteristics and regularity of electromagnetic waves. Huang Daqing et al. [27] analyzed and tailored the index of conduction and radiation test items in the National Military Standard. Song Huixuan et al. ${ }^{[28]}$ established the transmission line model of the cable harness. The surface current distribution of the cable harness was calculated by using the multi-conductor transmission line model. Zhang Dongxiao et al. ${ }^{[29]}$ took a certain type of unmanned target aircraft as the experimental object, built the test system by using the simulator construction method, put forward the radiation effect evaluation index and designed the dynamic monitoring system. Li Hongxiang ${ }^{[30]}$ designed a test method to test the radiation disturbance and radio frequency magnetic field radiation immunity of UAV products, and built two test equipments.

\subsubsection{Electromagnetic Conduction Test}

Conducted interference can be divided into capacitance conduction coupling, resistance conduction coupling and inductance conduction coupling. Huang Daqing et al. ${ }^{\text {[27] }}$ measured the conductive emission of the input power line of the tested equipment, aiming at the theoretical analysis and tailoring of the conductive emission CE101 of the power line, the theoretical analysis and tailoring of the conductive emission CE102 of the power line, and the theoretical analysis and tailoring of the conductive sensitivity CS101. Zhao Limin [31] deduced the INS heating model by system identification method, which provided a model basis for conduction interference analysis. Xu Ming et al. ${ }^{[32]}$ conducted conduction sensitivity analysis and design on the basis of functional design. The improved power filter, RS232, PCB chip and cable design circuit or schematic diagram were given.

\subsection{EMC design of UAV system}

System-wide EMC design is to release EMC design indexes of equipment and subsystem according to the estimated electromagnetic environment. In recent years, some developed countries have gradually developed to the stage of EMC system design. The United States has the largest anechoic chamber, reverberation chamber and nuclear electromagnetic pulse test site in the world, as well as test equipment with high test accuracy, wide frequency band and high power. Rheinland $\mathrm{T} \& \mathrm{~V}$ group has developed a large-scale industrial system-wide EMC testing equipment, which can be used for field testing of large-scale industrial machinery that can not be tested by ordinary EMC laboratories. TDK company of Japan built a large-scale high-performance anechoic chamber in Chiba in 2009, which can carry out EMC test, certification and rectification test of large-scale system [33].

At present, the consistency of system-wide EMC measurement in China is far less than that of equipment level EMC measurement, and the formulation of systemwide EMC test and corresponding standards is relatively lagging behind, so far there is no unified system-wide EMC test standard. The EMC laboratory of Beihang University has been able to undertake a variety of largescale scientific research tasks, such as the design and evaluation of full aircraft EMC of special aircraft, the integration of EMC test system, EMC test, solutions to EMC problems and the design of Airborne special antenna ${ }^{[34]}$. First Academy of aerospace has the first laboratory in China that can carry out the system-wide EMC test capability of launch vehicle ${ }^{[35] .}$ The EMC laboratory of AVIC helicopter design and Research Institute has passed the CNAs certification, and has the ability to undertake the EMC test of airborne equipment and subsystems, as well as the whole helicopter EMC test. Shanxi Haitai Electronic Co., Ltd. has developed a system-wide EMC automatic test system, which has the functions of electromagnetic environment measurement, system antenna coupling measurement, EMC safety margin measurement, power EMC characteristic test, etc.

\section{Key technical issues}

\subsection{Consistency between heaven and earth in EMC}

At present, most of the EMC characteristics of UAV systems are evaluated by matching test and comprehensive test. However, the test only examines the functions of wireless transceiver devices in different states of UAV, which can not meet the requirements of Heaven-Earth consistency instead of EMC test. In the course of flight, the electromagnetic environment of UAV is constantly changing. The equipment on the UAV is facing the electromagnetic environment impact caused by the high-power ground wireless equipment, and the electromagnetic environment caused by the wireless equipment in the aircraft and the target. Only by establishing an EMC test platform which is consistent 
with the actual flight environment, can it be possible to achieve the consistency between heaven and earth in the EMC test of UAV.

\subsection{Evaluation and criteria of UAV electromagnetic compatibility}

The most direct way to evaluate the electromagnetic compatibility of UAV system is the electromagnetic compatibility test. However, the test cycle is long and the cost is high, and the change of system equipment cannot be predicted. It is also difficult to carry out real-time inspection and evaluation of UAV electromagnetic environment in the flight process, resulting in the test data inconsistent with the actual situation. Therefore, it is necessary to develop reasonable EMC evaluation criteria to guide the design of UAV EMC.

\subsection{UAV system level EMC design}

EMC design of aircraft system includes data collection, model building, test verification, etc. in engineering development, it is often unable to carry out complete prediction and analysis due to schedule problems. After research and analysis, the author thinks that to carry out system level EMC design, we should first find out the weak link of UAV system design, and provide preliminary basis for EMC test; then combine comprehensive test to build the electromagnetic environment of UAV in flight; finally, carry out system EMC test to verify the coordination of wireless channel in dynamic electromagnetic environment Coordination and compatibility between man-machine and ground wireless equipment and electromagnetic compatibility of UAV system.

\subsection{EMC Intelligent Design Technology}

At present, the EMC analysis of UAV and other aircraft mainly depends on manual work. How to automatically realize the EMC test and evaluation needs the support of EMC expert system. The rapid development of artificial intelligence technology is widely used in robots, UAVs and other high-tech fields, but the EMC test system with artificial intelligence has not yet been reported. It is believed that EMC intelligent technology will be applied to UAV system design in the near future.

\section{Summary}

In recent years, the research on electromagnetic compatibility of UAV system at home and abroad has achieved fruitful results. However, the general evaluation criteria of UAV electromagnetic compatibility have not been formulated yet, and the existing criteria can not be used to guide the development of UAV models; Matching test and comprehensive test only examine the functions of wireless transceiver devices in different states of UAV, which can not meet the requirements of Heaven-Earth consistency instead of EMC test; the lack of a unified system level electromagnetic compatibility test standard in China makes it increasingly urgent to carry out relevant research work. With the increasing application of UAV, the research on electromagnetic compatibility of UAV system will attract more and more attention of aviation industry.

Now, deep research about antenna design and airborne antenna layout are carried out in our working group. In the future, we will do a lot of research on the anti-interference performance of data link system and the electromagnetic compatibility of UAV system.

\section{References}

1. Cai Rengang. EMC Principle, Design and Prediction Technology [M]. Beijing University of Aeronautics and Astronautics Press, 1997.

2. Gao Yougang. General Introduction to EMC [M]. Beijing University of Posts and Telecommunications Press, 2001.

3. Lu Yuzhong. Research on EMI Coupling and Protection of Small Aircraft in Complex Electromagnetic Environment [D], Xi'an University of Electronic Science and Technology, 2013.

4. Song Zuxun, Yu Bianzhang. Analysis of Electromagnetic Environment Effect of UAV System [J]. System Engineering and Electronic Technology, 2003; Volume 25, pp. 1108-1112.

5. Zhu Zhiyu, Zhang Bing, Liu Weiting. Application of Discrete Wavelet Filter in EMC Test of Aircraft [J]. Journal of Detection and Control. 2006; Volume 28, pp. 57-60.

6. Li Bo, Huang Daqing. Establishment of System Level Electromagnetic Radiation Index of UAV based on Electromagnetic Environment Effect [J]. Journal of Wuhan University (Science Edition). 2007; Volume 53, pp. 347-350.

7. Zhang Yong, Liu Junwei, Li Chao, etc. Research on Evaluation Index System and Application of Ship Electromagnetic Compatibility [J]. Ship Science and Technology. 2014; Volume 36, pp. 103-107.

8. Li Xiaojian, Kang Rui, Dai Fei, etc. Research on EMC Analysis Method based on Reliability Mathematics [J]. Telemetry and Telecontrol. 2010; Volume 31, pp. 66-70.

9. Tan Yuanyang. Analysis of scattering characteristics (cylindrical part) of aircraft antenna by UTD method [D]. Xi'an University of Electronic Science and Technology. 2004.

10. Yuan Jun. Research on high performance numerical algorithms for electromagnetic scattering and EMC analysis [D]. Xi'an University of Electronic Science and Technology. 2008.

11. Chen Jinji. Aircraft EMC prediction and simulation [D]. Xi'an University of Electronic Science and Technology. 2013.

12. Peng Tianhao. Research on the theory and key equipment of the METRE-WAVE broadband plasma antenna [M]. Shanghai Jiaotong University. 2015.

13. Liu Baihan. Research on numerical simulation technology for radio frequency compatibility of 
aircraft $[\mathrm{M}]$. Shenyang University of Aeronautics and Astronautics. 2017.

14. Han Xuefeng, Li Dong. Electromagnetic simulation analysis of airborne antenna based on finite difference time domain method [J]. Communication technology. 2017, 50 (11): 2472-2477.

15. Li Wei, Wei Guanghui, Pan Xiaodong et al. Prediction method of electromagnetic radiation effects of dual-frequency continuous wave in typical communication equipment band [J]. Systems engineering and electronic technology, 2016, 38 (11): 2474-2480

16. Guan Weiwei, Zhu Qianwang, Xu Jianzhong. Antijamming Analysis of UAV Data Link Based on SIR Equation [J]. Ship Electronic Engineering, 2013, 33 (1): $73-75$

17. Jiao Yanwei, Hou Deting, Zhou Dongfang et al. Evaluation efficiency of UAV in complex electromagnetic environment [J]. Intensive laser and particle beam, 2014, 26 (7): 11-16

18. Liu Ruiqi, Yao Zhicheng, Yang Jian, etc. [J]. Study on Start-up Strategy of Tracking Jamming for UAV Remote Control Link [J]. Electro-optic and Control. 2018, 25 (4): 25-27.

19. Guo Hongjing. Research on interference perception and recognition technology of UAV TT\&C link [D]. University of Electronic Science and Technology. 2014.

20. Zhang Xiaodong, Chen Ya, Xiao Xuerong. Research on EMI mechanism of main remote control data link of UAV [J]. Journal of Microwave Science. 2016, 32 (2): 90-96.

21. Guo Shuxia, Dong Zhongyao, Hu Zhantao, et al. Simulation of dynamic EMI environment for unmanned aerial vehicle data link[J]. EMC Design and its Application. 2013(8): 19-27.

22. Shen Yanan. Simulation study on electromagnetic environment adaptability of UAV remote control data link [J]. Value Engineering. 2012 (5): 178-179.

23. Song Zuxun, Yu Bianzhang. Adaptability of UAV data link to radar EMI [J]. Systems Engineering and Electronic Technology. 2004, 26 (12): 1790-1793.

24. Jiangdan. Research on electromagnetic conduction and radiation characteristics and suppression methods of multi-electric aircraft system [D]. Nanjing University of Aeronautics and Astronautics, 2016.

25. Wang Chao. EMI and Airworthiness of aircraft EWIS wiring [D]. Civil Aviation University of China. 2012.

26. Tan Yujian. Research on EMC analysis method for complex systems [D]. National University of Defense Science and Technology. 2009.

27. Huang Daqing, Li Bo. EMC Conduction and Radiation Test Project Cutting Method for UAV [J]. Optical Precision Engineering. 2009, 17 (2): 380-387.

28. Song Huixuan, Wu Gang, Zhu Long, etc. EMC analysis of radiation from cables and cables harness inside aircraft [R]. National Conference on Microwave and Millimeter Wave. 2013: 1532-1535.

29. Zhang Dongxiao, Chen Ya, Cheng Erwei, et al. Experimental study on continuous wave electromagnetic radiation effects of an unmanned target aircraft [J]. Journal of Hebei Normal University (Natural Science Edition). 2017, 41 (1): 39-44.

30. Li Hongxiang. Testing of Radiation Disturbance and Radiation Immunity of UAV [D]. Heilongjiang University. 2017.

31. Zhao Limin. Research on Conducted EMI Analysis Method for Aircraft Electrical System [D]. Harbin Engineering University. 2012.

32. Xu Ming, Chen Yan. Conduction sensitivity analysis and design of an airborne flight controller [J]. Ship electronics engineering. 2016.

33. Zhu Chengbang, Hua Yang. Ship Technology Process Elements and Electromagnetic Compatibility Test[J]. Ship Electronic Engineering, 2013; Volume 33, pp. 171-173.

34. Sun Guangjing. Progress the development of ship EMC Technology [J]. Ship Electronic Engineering. 2007; Volume 27, pp. 20-22, pp. 40.

35. Lai Shunxiang, Yang Huiqin. Discussion on EMC Test Method of Space Launch Site [J]. Ship Electronic Engineering. 2004; Volume 24, pp. 160162. 